\title{
Experimental Investigation of Split and Recombination Micromixer in Confront with Basic T- and O- type Micromixers
}

\author{
Mohammad Nimafar*, Vladimir Viktorov, Matteo Martinelli \\ Department of Mechanical and Aerospace Engineering, Politecnico di Torino, Turin, 10129, Italy
}

\begin{abstract}
The paper presents an experimental comparison of three types of static micromixer. Efficiencies of a split and recomb ination micromixer (SAR) based on plate symmetrical modules (PSM) and basic T-type and O-type mixers are examined. Experimental tests were carried out in the laminar flow regime with a low Reynolds number range, $0.083 \leq \operatorname{Re} \leq$ 4.166 and image-based techniques were used to evaluate mixing efficiency. Experimental results illustrate that the micro mixers with splitting and recombination have outstanding mixing efficiency than those of without SAR process. Indeed split and recombine (SAR) structures of the flow channels result in the reduction of the diffusion distance of two fluids and optimize the diffusion process and after a short distance from inlet high mixing efficiency can be achieved. Also, experimental data show that the SAR PSM mixer is up to 99\% efficient, and that efficiency reaches $90 \%$ in a short distance, demonstrating this type of mixer's high mixing performance and the effect of splitting and recombination on the degree of mixing and the efficiency of the micromixer.
\end{abstract}

Keywords Gray Scale Value, Image-Based Technique, MEMS, Microfluidic, Passive Micromixer, SAR Process

\section{Introduction}

Developing devices for microfluidic technology has been a major concern for industry in the past decade. Microfluidic devices offer many advantages over conventional techniques, including reduced reagent consumption, low energy requirements, faster analysis and low cost. Micromixers are key elements in microfluidic technology and have been addressed by a large body of research. In general, micromixers can be classified as either active and passive fluid mixers[1-4], depending on how they manipulate the fluids to be mixed. Active micromixers require external forces, such as acoustically induced vibrations, electrokinetic instabilities, periodical variation of pumping capacity, electrowetting-induced merging of droplets, magneto-hydrodynamic action, small impellers, piezoelectrically vibrating membranes or integrated micro valves and pumps[1-15]. By contrast with active mixers, a passive type requires no external energy and agitation, except that associated with producing the required pressure drop for flow. In a typical microfluidic device, the flow is laminar and characterized by low Reynolds numbers. The

* Corresponding author:

mohammad.nimafar@polito.it (Mohammad Nimafar)

Published online at http://journal.sapub.org/mechanics

Copyright (C) 2012 Scientific \& Academic Publishing. All Rights Reserved mixing process is slow and mainly due to molecular diffusion. The hydrostatic potential is used to restructure a flow in a way which results in faster mixing[1-3]. The mixing of mic rofluids in a passive device is based on several main principles: a) flow lamination, which is used in basic T-mixers and Y- mixers[16-21], in mixers with different geometries: zig-zag, square-wave, rho mbic and the like[2223], and in serial mu lti-stage and mu lti-layer mixers [24-26]); b) chaotic mixing by eddy formation, stretching and folding[27-33]; c)split-and-recombine concepts (SAR)[34-39]. The mixing performance of passive mixers can be enhanced by extending the contact interface of fluids and by improving the transverse components of flow movement, thus decreasing the length and duration of mixing[37]. Previous investigations[34-39] have demonstrated that devices with a SAR mechanism increase the contact interface of fluids to generate rapid and homogeneous mixing that is suitable for slow laminar fluid flow. At the same time, some of the previous SAR mixers are complicated in terms of design and construction because of their three-dimensional micro structure[34],[35],[37]. The mixing mechanism of the mixer described in[38] is based on splitting and recombining (SAR) with chaotic advection and is best suited for mixing fluids with relatively high Reynolds numbers. The $\sigma$-type plane static mixer with splitting and inverse recombination[36] is simple in structure, but the branched channels of the $\sigma$-type plane module are not identical or 
symmetric. The channels vary in form and length, resulting in varied channel conductance.

In this article we compare the efficiencies of split and recombination micromixer (SAR) based on plate symmetrical modules (PSM) and basic T-type and O-type mixers. Results are presented for experimental tests carried out in the laminar flow regime with a low Reynolds number range, $0.083 \leq \operatorname{Re} \leq 4.166$. Image-based techniques were used to evaluate mixing efficiency. Two colored water solutions (blue and yellow) are used for mixing.

\section{Micromixer Design}

On the basis of the investigation presented in[39], a split and recombination micromixer consisting of plate symmetrical modules (SA R PSM micro mixer) was designed and constructed with polycarbonate using a computerized milling process. SAR PSM micromixer design is shown in Figures 1 and 2.

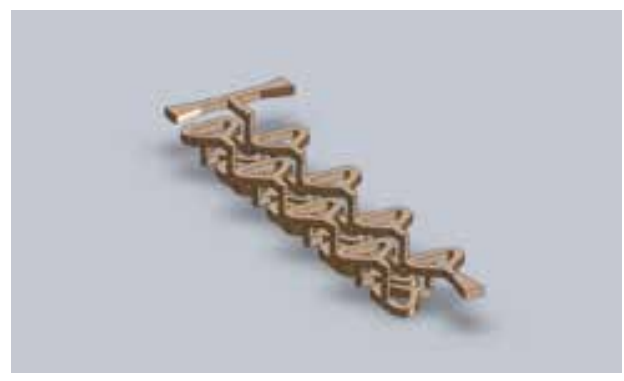

Figure 1. Design of a SAR PSM micromixer

Channel width and height are $0.4 \mathrm{~mm}$. As can be seen from Figure 2, the SA R PSM mixer has a two-layer structure. The modules of the upper layer (shown in yellow) and the modules of the lower layer (red) are connected by simple vertical channels (red). The inlet to the mixer is designed as a T-junction element (left side of figure).



Figure 2. SAR PSM micromixer

The principal plate modules of the mixer have the same symmetrical shape and are designated as the plate symmetrical module (PSM).

Figure 3 shows structural details of the SAR PMS micromixer. Two miscible liquids move through the entrance and reach the mixing region of the T-junction element, where they flow along the $+\mathrm{X}$ direction. The fluids flow in parallel through the first element. The stream then reaches the vertical channel (segment 2) and moves in the $-\mathrm{Z}$ direction. At the end of the vertical channel, the stream reaches the first $\mathrm{Y}$ segment (principal module) and is split into two flows (segment 3 ).
One strea m moves in the $+\mathrm{X}$ direction and the other moves in the $-\mathrm{X}$ direction. Both flows then cross the $\mathrm{Y}$ segment of the principal module, at the end of which they are recomb ined and move in the $-Y$ direction. Subsequently, the reunited stream reaches the verticalchannel and moves in the $+\mathrm{Z}$ direction. At the end of the vertical channel, it arrives at the Ysegment and is again split into two streams. One stream moves in the $+Y$ direction and the other in the $-\mathrm{Y}$ direction, and both then pass through the Y segment. At the end of the $\mathrm{Y}$ segment, they are once again recombined and move in the $+\mathrm{X}$ direction. This process continues to the end of the mixer microchannels.

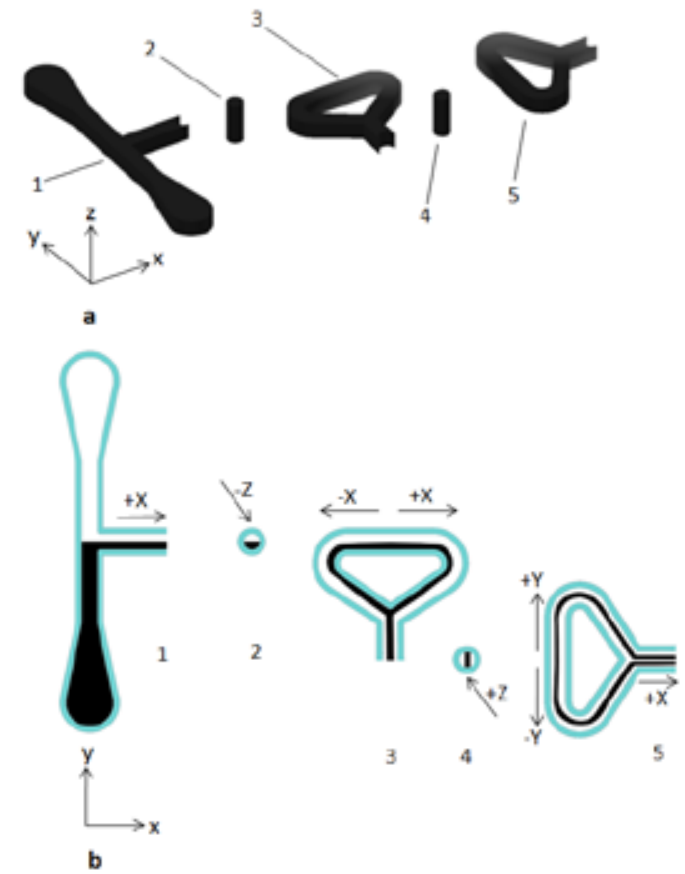

Figure 3. SAR PSM micromixer struct ural details; a) PSM microchannel segments; b) Sequential lamination of layers

As can be readily seen, this method of splitting and recombining the flow provides a way of increasing the number of fluid layers.

The T-mic romixer and O-mixer are illustrated in Figure 4. The T-micromixer is the simplest microfluidic device and consist of two inlets with a straight channel.



Figure 4. (a) T-micromixer (b) O-micromixer 
Figure 4b illustrates the O-mixer[40], whose microchannel is divided into two seg ments: straight channel and O-segment. Two species flow into the microchannel and through the straight section. Subsequently, the stream is divided into two parts and the two streams connect to each other at the end of the O-segment. This splitting and rejoining induces the molecular diffusion effect to enhance mixing efficiency. This process takes place six times along the microchannel.

Both of the T- and O-type micromixers are designed and fabricated from plexig las using a computer milling process.

\section{Materials and Method}

The test bench layout used for this experimental investigation is shown in Figure 5.

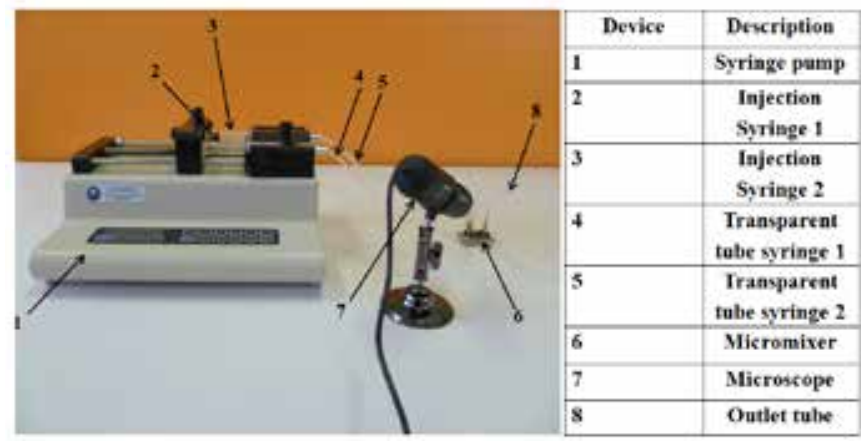

Figure 5. Test bench layout

Microscope: The microscope used during the mixing process is a Veho model VMS-004D-400X USB microscope, with 400X magnification, 2 Megapixel Cmos lens (interpolated) and measurement software.

Syringe pump: Flow rate was measured by means of a syringe pump. The syringe pump used in this study is a KDS 210 series. The flow rate range for this instrument is $0.1 \mu 1 / \mathrm{hr}$ (10 $\mu$ l syringe) to $506 \mathrm{ml} / \mathrm{hr}(60 \mathrm{ml}$ syringe $)$.

\section{Experimental Procedure}

Experimental tests were performed under the microscope (VMS-004D, Veho) with high speed image acquisition. As miscible fluids, colored water (blue and yellow) was used in all tests on the three micromixers. Colored water solutions for the tests were p roduced by blending 1 gram powder in 0.5 liter water. Species flow in the microchannel was controlled by means of a programmable syringe pump. Figure 6 depicts the mixing process through the microchannels in $\mathrm{T}, \mathrm{O}$ and SAR PSM micromixers. After the mixing process was visualized as shown in the figure, mixing efficiencies for all micromixers were calculated and compared. As Figure 7 shows, two samples are concentrated into one stream while fluid flows.

During the mixing process, several pictures were taken with the microscope from the in let section to the end of the channel. This was repeated for all Reynolds numbers used in this study.

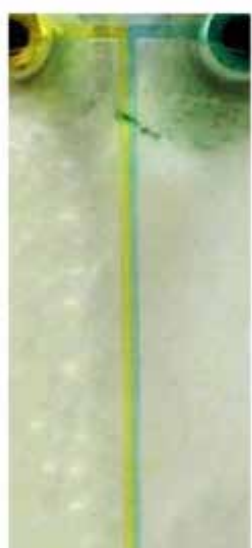

a

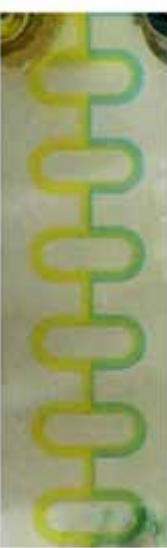

b

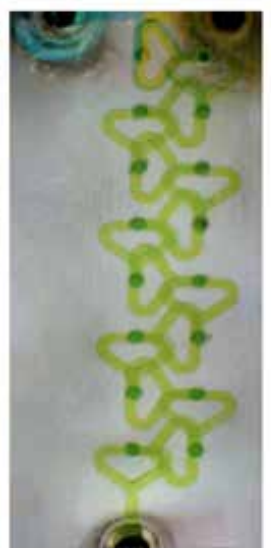

$\mathrm{c}$
Figure 6. Micromixers after filling: (a) T-micromixer (b) O-micromixer (c) SAR PSM micromixer

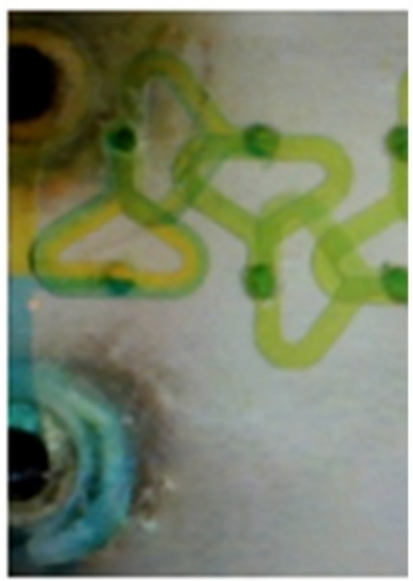

a

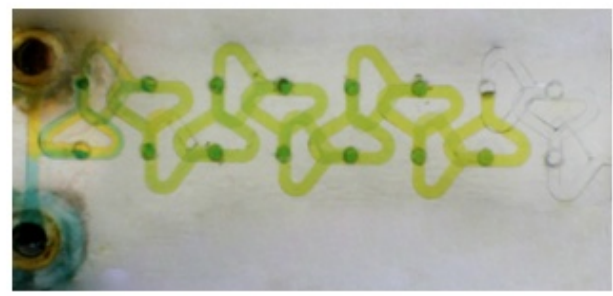

b

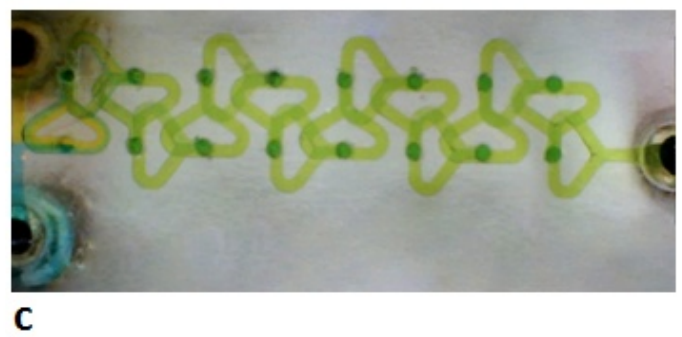

Figure 7. SAR PSM micromixer after filling

To investigate the mixing process, experimental tests were performed at five flow rates as shown in Table 1 : 
Table 1. Flow rate and $R e$ values during experimentaltests

\begin{tabular}{|c|c|c|c|}
\hline $\begin{array}{c}\text { Flow Inlet } \\
(\mathrm{ml} / \mathrm{min})\end{array}$ & $\begin{array}{c}\text { Mass Flow } \\
(\mathrm{kg} / \mathrm{s})\end{array}$ & $\mathrm{V}(\mathrm{m} / \mathrm{s})$ & $R e$ \\
\hline 0.001 & $1.66 * 10^{8}$ & 0.0001 & 0.083 \\
\hline 0.005 & $8.31 * 10^{8}$ & 0.0005 & 0.416 \\
\hline 0.01 & $1.66^{*} 10^{-7}$ & 0.0010 & 0.832 \\
\hline 0.02 & $3.32 * 10^{-7}$ & 0.0020 & 1.666 \\
\hline 0.05 & $8.32 * 10^{-7}$ & 0.0052 & 4.166 \\
\hline
\end{tabular}

Image-based techniques were used to evaluate the mixing performance of the prototypes, and the gray scale value was applied for post processing. To analyze the image, the captured color images were converted into gray scale images. Gray scale images are also called monochromatic, denoting the absence of any chro matic variation (also called bi level or binary images). To convert any color to a gray scale representation of its luminance, the value of its red, green and blue (RGB) primaries must first be obtained in linear intensity encoding, after which $30 \%$ of the red values, 59\% of the green values, and $11 \%$ of the blue values are added together. This technique (gray scale value) is useful for highlighting regions of interest, especially the concentration field in the mic ro mixer[41].

\section{Post Processing}

To evaluate the standard deviation of concentration, mixing index and efficiency of the prototypes, three kinds of Matlab program code are written (PDF1, PDF2 and Efficiency). Data was analyzed using the following procedure:

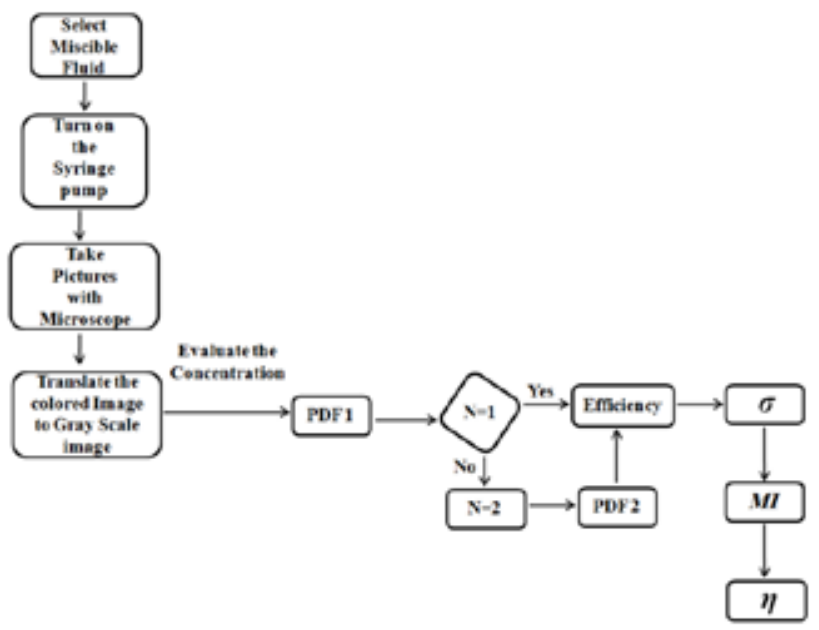

Figure 8. Flow chart for experimental analyzing

Figure 9 presents the probability distribution function (PDF) in terms of concentration. Subsequently, probabilities for different concentration values and for various Reynolds numbers were calculated and plotted for the SAR PSM prototype.
In a region such as the inlet or other regions where two species are not mixed, there are two predominant concentration values of 0 and 1 , resulting in two separate peaks. In a region of well-mixed fluids, there is one predominant concentration resulting in a single peak.

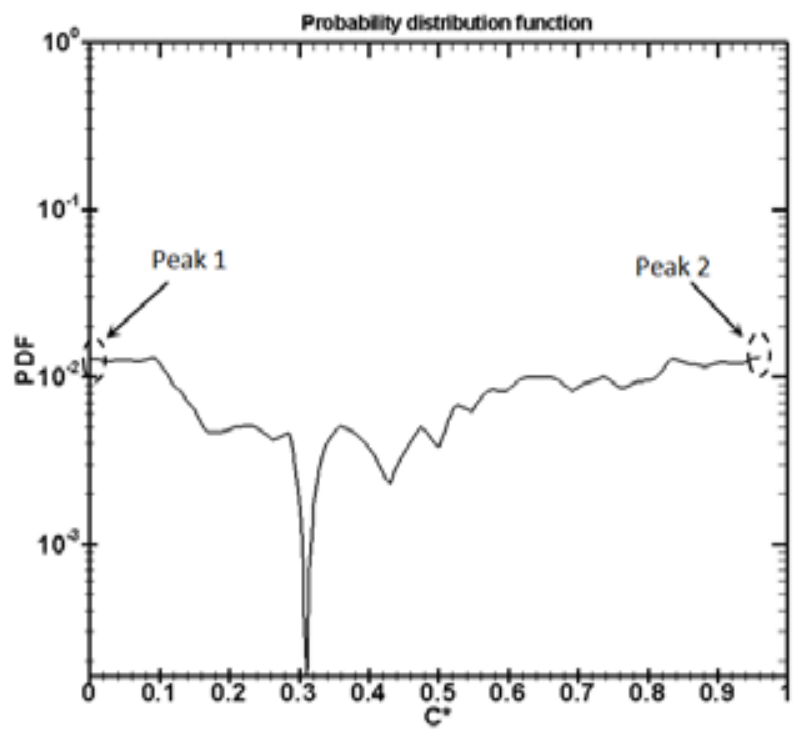

Figure 9. PDF in SAR P SM micromixer, $\mathrm{Re}=1.666$, entrance region

The homogeneity can be represented as the standard deviation of the concentration :

$$
\sigma=\sqrt{\frac{1}{N} \sum_{I=1}^{N}\left(C_{i}^{*}-\overline{C^{*}}\right)^{2}}
$$

where $C^{*}$ is the normalized concentration and $C \square^{*}$ is the expected normalized concentration. Also the standard deviation is 0 for well-mixed cases. The standard deviation can be normalized again by the mean concentration to evaluating the mixing index (MI) :

$$
M I=\sqrt{\frac{1}{N} \sum_{I=1}^{N}\left(\frac{C_{i}^{*}-\overline{C^{*}}}{\overline{C^{*}}}\right)^{2}}
$$

The mixing indexes (MI) are 1 and 0 for the unmixed case and well-mixed case, respectively. Mixing efficiency can be defined through equation 3 :

$$
\eta=1-M I=1-\sqrt{\frac{1}{N} \sum_{I=1}^{N}\left(\frac{C_{i}^{*}-\overline{C^{*}}}{\overline{C^{*}}}\right)^{2}}
$$

If the two species are fully mixed, mixing efficiency is $1(100 \%)$. An efficiency between around $80 \%$ and $100 \%$ is acceptable for mixing process applications[41], and useful for many applications. As shown in Figure 10, analys is of the experimental data indicates that at the starting point where two fluids join together, the standard deviation is 0.5 , mixing index is 1 and efficiency is $0 \%$.

The experimental results demonstrate that efficiency is 98\%at the end section of the SAR PSM mixer under certain conditions. For example, Figure 11 illustrates the SAR PSM mixer's concentration, while Figure 12 shows $\sigma$, MI and $\eta$ at 
the same Reynolds number of 1.666 , where efficiency is 98\%. These processes continue for Reynolds numbers of $0.083,0.416,0.832$ and 4.166 for the SAR PSM prototype.
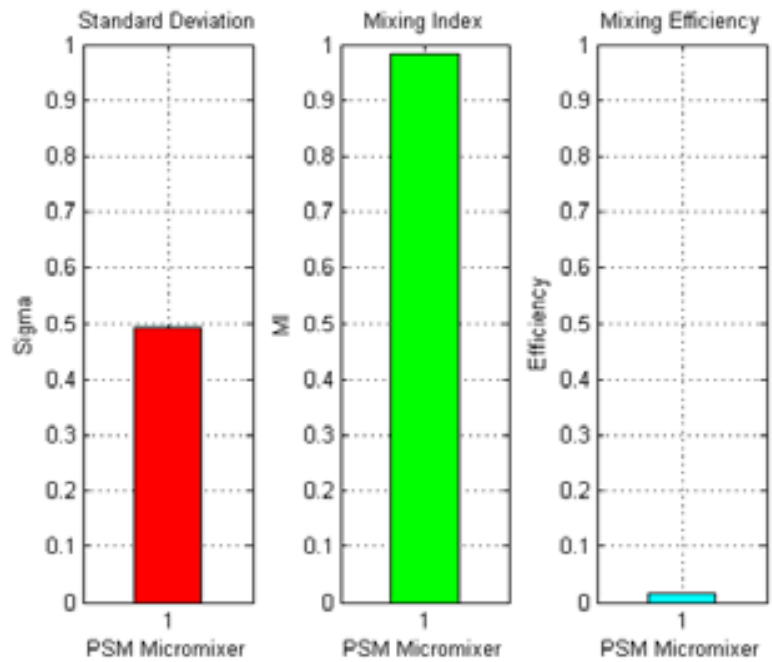

Figure 10. $\sigma, M I$ and $\eta$ in SAR PSM micromixer, $\mathrm{Re}=1.666$, inlet section



Figure 11. PDF in SAR PSM micromixer, $R e=1.666$, after $20 \mathrm{~mm}$

Concentration based analysis is the most reputable and trustworthy method of investigating and evaluating mixing efficiency.

By improving concentration image analysis, the detailed process taking place in the microchannel can be investigated. When flow is not in the turbulent regime, it is essential to observe changes in concentration through the channel to understand mixing performance. Once the concentration field has been determined with the probability distribution function in terms of concentration, then according to equations 1, 2 and 3 , the standard deviation of the concentrations, mixing index and efficiency can be calculated (and plotted).
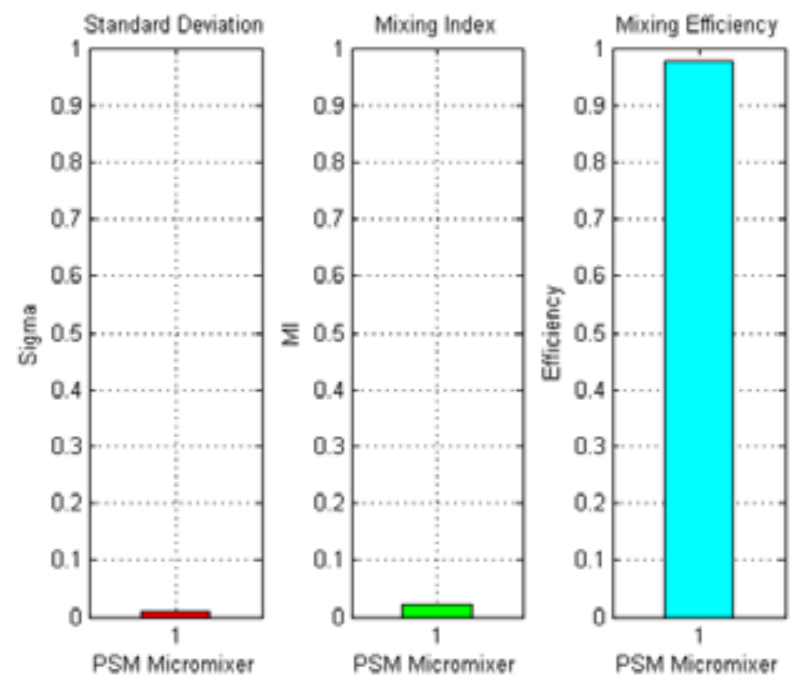

Figure 12. $\sigma, M I$ and $\eta$ in SAR P SM micromixer, $\mathrm{Re}=1.666$, after $20 \mathrm{~mm}$

\section{Result and Discussion}

At low Reynolds numbers, mixing takes place through molecular diffusion alone. Results obtained for the PSM micro mixer are compared with those for the T- and O-mixers at the same Reynolds number in Figure 13.

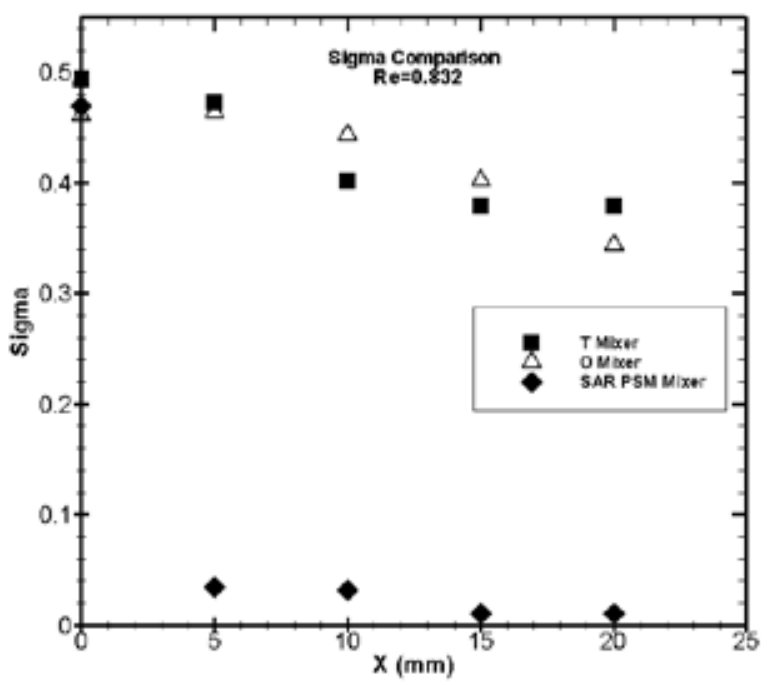

Figure 13. Comparison of $\sigma$ for $\mathrm{T}, \mathrm{O}$ and SAR PSM mixers at $\mathrm{Re}=0.832$

For the SAR PSM mixer, standard deviation of the concentration changes fro $\mathrm{m} 0.5$ to 0.01 at $\operatorname{Re}=0.832 ; \sigma=0.01$ at the ending region is a good result for the mixing process. For the O-mixer and T-mixer, standard deviation of the concentration at the same Reynolds number is 0.343 and 0.378 respectively. Comparing the standard deviation indicates that mixing is more complete in the SAR PSM mixer than in the O- and T-mixers.

Figure 14 shows that at $\mathrm{Re}=0.832$ the mixing index is 0.016 and 0.68 in the PSM mixer and the O-mixer respectively. Figure 14 also shows that MI decreases in the first five millimeters of the microchannel from the in let section at a faster rate in the PSM mixer than in the O- and 
T-mixers, and drops below 0.2. This characteristic is significant for micro mixer design, as it illustrates that mixing is fully acco mplished in a short distance.

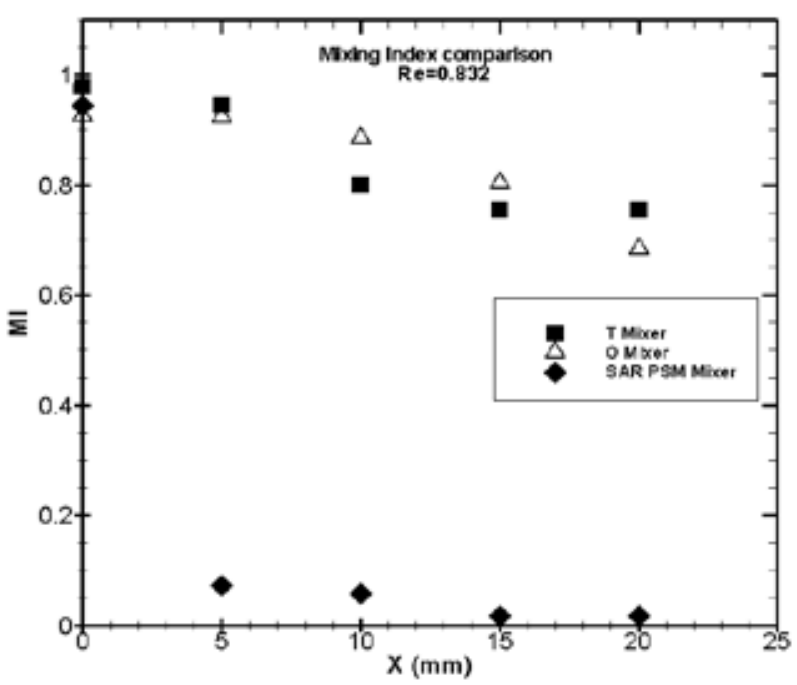

Figure 14. Comparison of $\mathrm{MI}$ at $\mathrm{Re}=0.832$

During tests at other Reynolds numbers, it was found that the mixing index increases along with the Reynolds number. As shown in Figure 14, however, the SAR PSM mixer's MI is less than 0.1 at all tested flow rates, indicating that mixing is accomplished effectively at a range of flow rates $(0.001 \leq$ $\mathrm{Q} \leq 0.05$ ).

Results of experimental tests on the SAR PSM micro mixer are illustrated in this paragraph.



Figure 15. Comparison of $\eta$ at $\operatorname{Re}=0.832$

Figure 15 compares the efficiency of SAR PSM, T- and O-micromixers at $R e=0.832$. As can be seen, the SAR PSM mixer is more efficient than the other prototypes in all regions of interest.

Figure 16 also shows that the T-and O-mixer's efficiency is $9 \%$ and $13 \%$ respectively, which is not acceptable for mixing processes, whereas the efficiency of the PSM mixer is $97 \%$.

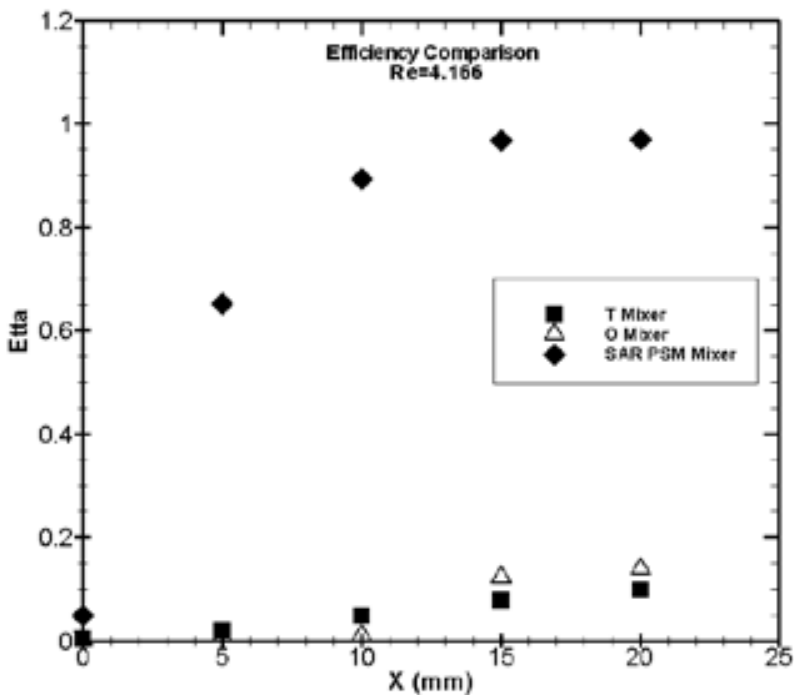

Figure 16. Comparison of $\eta$ at $\operatorname{Re}=4.166$

A comparison of the SAR PSM mixer's efficiency at $\operatorname{Re}=4.166$ also indicates that fluids are well mixed at this Reynolds number, and that it is possible to increase the flow rate to rapidly achieve complete mixing.

This capability is significant for micromixer design, as this device requires special geometry since it cannot rely on turbulence flow to enhance mixing performance.

Figures 17-20 illustrate efficiency in terms of Reynolds number. As the diagrams show, efficiency drops as the Reynolds number increases, but the rate of decrease in the SAR PSM mixer is very slow and negligible after $5 \mathrm{~mm}$. In some micro mixers, efficiency is about $80 \%$ or mo re at $\operatorname{Re} \leq 1$, which is useful for mixing applications.

As the diagrams show, efficiency of the T- and O-mixers is less than $80 \%$ for $1 \leq \operatorname{Re} \leq 4.66$, which is not acceptable for mixing processes. In the SAR PSM mixer, however, the fluids are well mixed even for $1 \leq \mathrm{Re} \leq 4.66$. Rapid mixing is thus possible in this kind of geo metry, and complete mixing as seen to take place in this prototype.

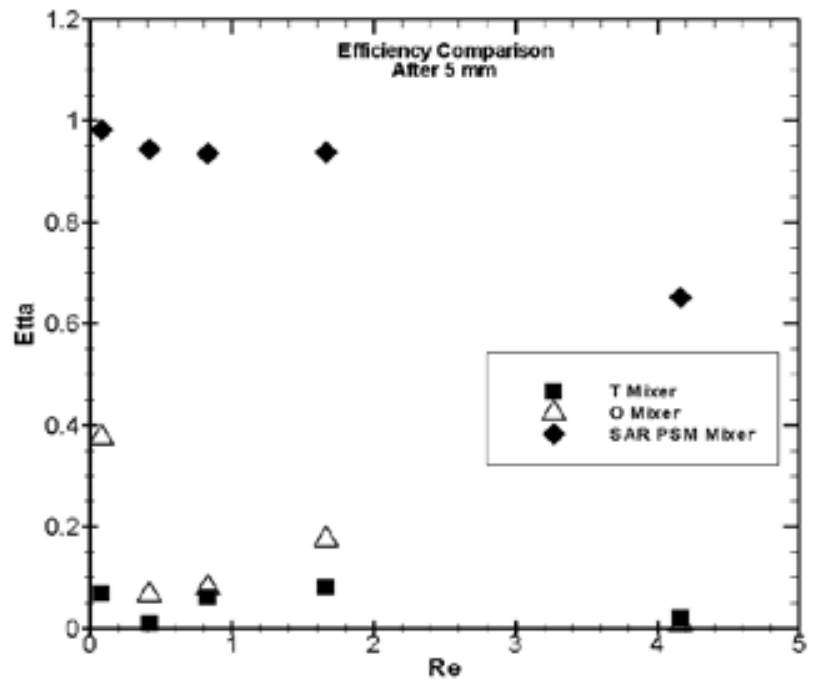

Figure 17. Comparison of $\eta$ in terms of Re, after $5 \mathrm{~mm}$ 


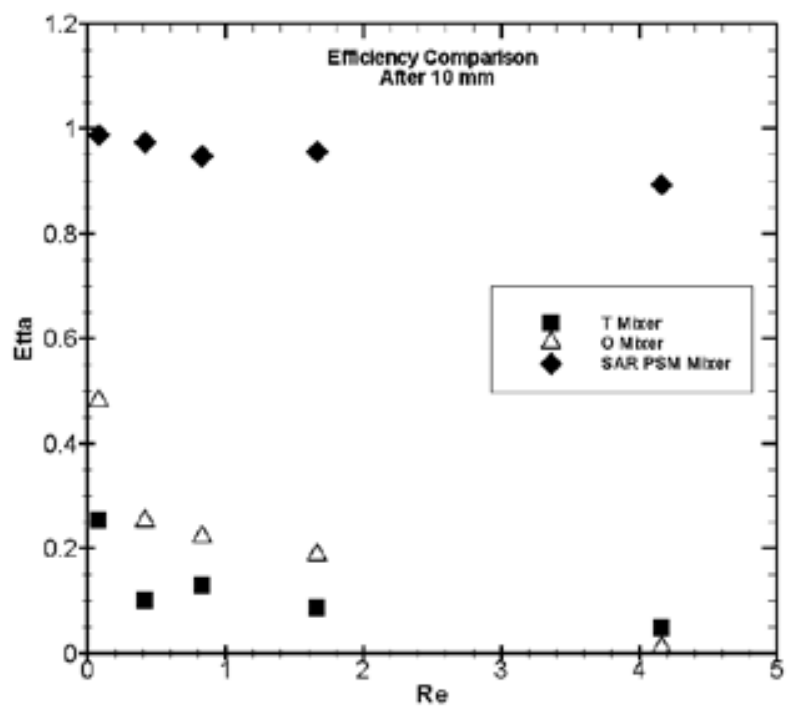

Figure 18. Comparison of $\eta$ in terms of Re, after $10 \mathrm{~mm}$

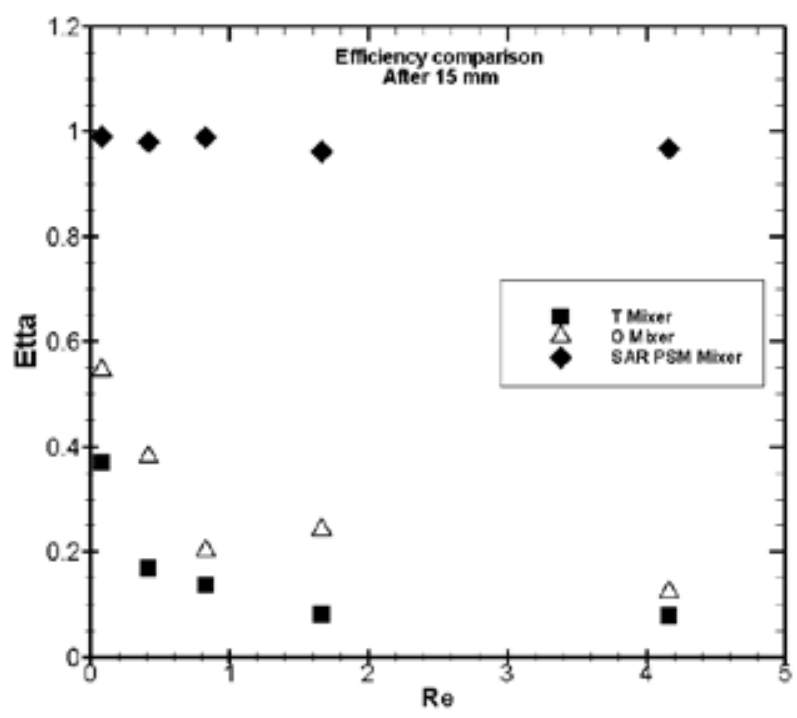

Figure 19. Comparison of $\eta$ in terms of Re, after $15 \mathrm{~mm}$

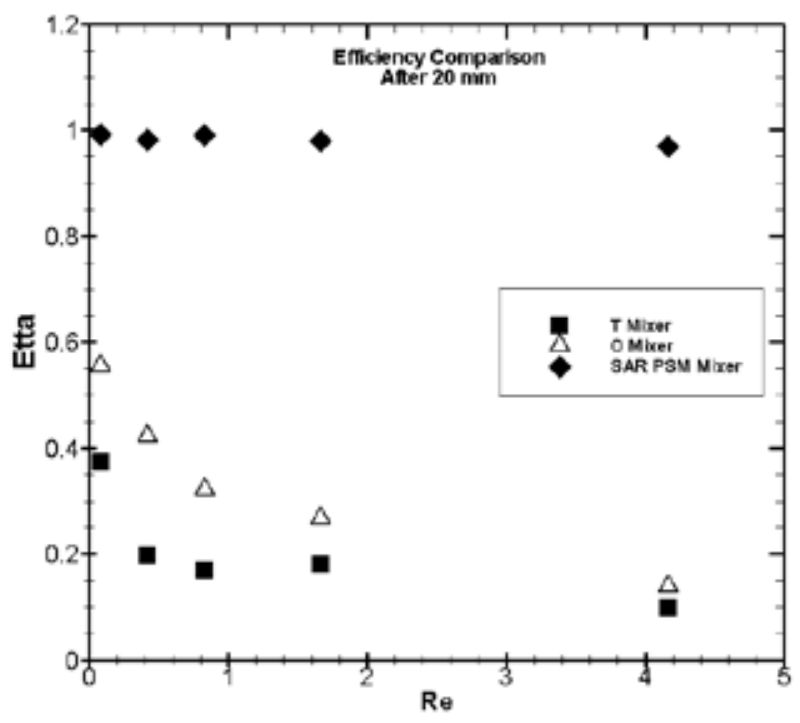

Figure 20. Comparison of $\eta$ in terms of Re, after $20 \mathrm{~mm}$
As Figure 20 shows, SAR PSM mixer efficiency is over 98\% and acceptable for mixing processes at all tested Reynolds numbers.

\section{Conclusions}

This paper explained a 3D splitting and recombination passive mic romixer called 'SAR PSM micromixer' which was designed and constructed with polycarbonate using a computerized milling process. Mixing characteristics of two species are analyzed via experimental investigation with various inlet flow rates (velocities) and results compared with the previous T-type and O-type well-known geo metries mixers which were designed and fabricated from plexiglas using a computer milling process.

In micromixers, viscosity dominates flow and mixing is limited to molecular diffusion due to the laminar nature of micro flows such as T- and O-type micromixers and because of low mixing performance in these kind of microchannels, they are not applicable in industries.

Since effective mixing is a crucial process in microfluidic systems, hence focus on this field of research is to propose a device itself and optimization of the geometry to help diffusion process to enhance fluid mixing within the microchannel to reach the desired degree of mixing.

Comparison between SAR PSM and other mixers demonstrated that the SAR PSM micromixer is more efficient for mixing processes at low Reynolds numbers ( $\mathrm{Re}$ $<5$ ), which generate complete mixing.

Experimental data show that the standard deviation of the concentration at the outlet of the SAR PSM micromixer is below 0.1 , which is less than that of the T- and O-mixers in the $0.083 \leq \operatorname{Re} \leq 4.166$ range.

The mixing index changes at a more rapid rate in the SAR PSM mixer than in the T- and O-mixers at all flow rates. It can be concluded that mixing is accomplished more quickly in the SAR PSM micromixer than in the other prototypes. As mentioned before, After short distance, SAR PSM mixer's efficiencies are acceptable while in other prototypes the degree of mixing is low demonstrating the effect of splitting and recombination on micromixer's efficiency. Also, when the flow rate increases, standard deviation of concentration and mixing index rise and efficiency drops. However, the rate of change in the SAR PSM mixer is very slow, and it was found that flow rate can be increased to rapidly achieve complete mixing in the passive micromixer.

\section{REFERENCES}

[1] V. Hessel, H. Löwe, F. Schönfeld , "Micromixers-a review on passive and active mixing principles", Chemical Engineering Science, 60 (2005) 2479 - 2501.

[2] E. A. Mansur, Y. Mingxing, W. Yundong, D.Youyuan, "A State-of-the-Art Review of Mixing in Microfluidic Mixers", 
Chinese Journal of Chemical Engineering, 16(4) (2008) 503-516.

[3] N.T. Nguyen , Z. Wu, "Micromixers-a review", J. Micromech. Microeng., 15 (2005) R1-R16.

[4] H. Bockhorn, D. Mewes, W. Peukert, H.-J.Warnecke, "Micro and Macro Mixing: Analysis, Simulation and Numerical Calculation (Heat and Mass Transfer)", Springer (2010) ISBN: 3642045480 , 348 pages.

[5] Z. Yang et al., "Ultrasonic micromixer for microfluidic systems", Sensors and Actuators A 93 (2001) 266-272.

[6] R.H. Liu et al., "Hybridization enhancement using cavitation microstreaming", Analytical Chemistry, 75 (8) (2003) 1911-1917.

[7] M.H. Oddy, J.G. Santiago, J.C. Mikkelsen, "Electrokinetic instability micromixing", Analytical Chemistry 73 (24)(2001) 5822-5832.

[8] I Glasgow, N. Aubry, "Enhancement of microfluidic mixing using time pulsing", Lab on a Chip 3 (2003) 114-120.

[9] X. Niu, Y.-K. Lee, "Efficient spatial-temporal chaotic mixing in micro-channels" Journal of Micromechanics and Microengineering 13 (2003) 454-462.

[10] S. Qian, H.H. Bau, "A chaotic electroosmotic stirrer", Analytical Chemistry 74 (15) (2002) 3616-3625.

[11] A. Neilda, T. W. Nga, G. J. Sheardb, et al., "Swirl mixing at microfluidic junctions due to low frequency side channel fluidic perturbation", Sensors and Actuators B 150 (2010) 811-818.

[12] P. Woias, K. Hauser, E. Yacoub-George (Eds.), "An active silicon micromixer for $\mu$-TAS applications" In: van den Berg, A., Olthuis, W., Bergveld, P. (Eds.) Micro Total Analysis Systems Kluwer Academic Publishers, Dordrecht (2000) pp. 277-282.

[13] J. Voldman, M.L. Gray, M.A. Schmidt, "Liquid mixing studies with an integrated mixer/valve" In: Harrison, J., van den Berg, A. (Eds.) Micro Total Analysis Systems, Kluwer Academic Publishers, Dordrecht (1998) pp. 181-184.

[14] L.S. Jang, S.H. Chao, M. R. Holl, D. R. Meldrum, "Resonant mode-hopping micromixing", Sensors and Actuators A, 138 (2007), 179-186.

[15] Y. Ma, C.P. Sun, M. Fields, Yang Li, et al, "An unsteady microfluidic T-form mixer perturbed by hydrodynamic pressure", J. Micromech. Microeng. 18 (2008) 045015 (14pp).

[16] A.E. Kamholz et al., "Quantitative analysis of molecular interactive in microfluidic channel: the T-sensor", Anal. Chem. 71 (1999) 5340-7.

[17] A.E. Kamholz and P. Yager, "Molecular diffusive scaling laws in pressure-driven microfluidic channels: deviation from one-dimensional Einstein approximations", Sensors and Actuators B 82 (2002) 117-121.

[18] R.F. Ismagilov et al., "Experimental and theoretical scaling laws for transverse diffusive broadening in two-phase laminar flows in microchannel", Appl. Phys. Lett., 76 (2000) 2376-2378.

[19] S. H. Wong, M.C.L. Ward, C.W. Wharton, "Micro T-mixer as a rapid mixing micromixer", Sensors and Actuators B 100 (2004) 359-379.

[20] S.P. Sullivan, B.S. Akpa et al., "Simulation of miscible diffusive mixing in microchannels", Sensors and Actuators B 123 (2007) 1142-1152.

[21] M.J. Swickratha, S.D. Burnsa, G.E. Wnekb, "Modulating passive micromixing in 2-D microfluidic devices via discontinuities in surface energy", Sensors and Actuators B 140 (2009) 656-662.

[22] S. Hossain, M.A. Ansari, K.Y. Kim, "Evaluation of the mixing performance of three passive micromixers", Chemical Engineering Journal 150 (2009) 492-501.

[23] C. K. Chung, T. R. Shih, "Effect of geometry on fluid mixing of the rhombic micromixers", Microfluidics and Nanofluidics (2008) Volume 4, Number 5, 419-425.

[24] J. Branebjerg, P. Gravesen, J.P. Krog, C.R. Nielsen, "Fast mixing by lamination", Proc. MEMS 96, 9th IEEE Int. Workshop MicroElectromechanical System (San Diego, CA) (1996) pp 441-446.

[25] M. S. Munson, P. Yager, "Simple quantitative optical method for monitoring the extent of mixing applied to a novel microfluidic mixer", Anal. Chim. Acta (2004) 507, 63-71.

[26] T. Tofteberg, M. Skolimowski, E. Andreassen, O. Geschke, "A novel passive micromixer: lamination in a planar channel system", Microfluidics and Nanofluidics (2010) Volume 8, Number 2, 209-215.

[27] T.N.T. Nguyen, M.C. Kimb, J.S Park, N.E. Lee, "An effective passive microfluidic mixer utilizing chaotic advection", Sensors and Actuators B, 132 (2008) 172-181.

[28] S. H. Wong, P. Bry ant, M. Ward, C. Wharton, "Investigation of mixing in a cross-shaped micromixer with static mixing elements for reaction kinetics studies", Sensors and Actuators B 95 (2003) 414-424.

[29] Z. Xua, C. Li, D. Vadilloc, X. Ruanb, X. Fub, "Numerical simulation on fluid mixing by effects of geometry in staggered oriented ridges micromixers", Sensors and Actuators B 153 (2011) 284-292.

[30] X. Fu, S. Liu , X. Ruan, H. Yang, "Research on staggered oriented ridges static micromixers", Sensors and Actuators B 114 (2006) 618-624.

[31] K. Malechaa, L.J. Golonkaa, J. Bałdygab et al., "Serpentine microfluidic mixer made in LTCC", Sensors and Actuators B 143 (2009) 400-413.

[32] Y. Du, Z. Zhang, C. Yim, M. Lin, X. Cao, "Evaluation of Floor-grooved Micromixers Using Concentration-channel Length Profiles", Micromachines (2010), 1, 19-33.

[33] T. R. Shih, C.K. Chung, "A high-efficiency planar micromixer with convection and diffusion mixing over a wide Reynolds number range", Microfluidic Nanofluidic (2008) 5:175-183.

[34] S.W. Lee, D.S. Kim, S.S. Lee, T.H. Kwon, "Split and recombination micromixer based on PDMS three-dimensional micro structure", The 13th International Conference on Solid-state Sensors, Actuators and Microsy stems, Seoul, Korea, June 5-9 (2005) 1533-1536.

[35] S.W. Lee, D.S. Kim, S.S. Lee, T.H. Kwon, "A split and 
recombination micromixer fabricated in a PDMS three-dimensional structure", J. Micromech. Microeng. 16 (2006) 1067-1072.

[36] K. Ohkawa, T. Nakamotob, Y. Izukab, et al., "Flow and mixing characteristics of $\sigma$-type plate static mixer with splitting and inverse recombination", Chemical Engineering Research and Design 86 (2008) 1447-1453.

[37] S.W. Lee, S. S. Lee, "Rotation effect in split and recombination micromixing", Sensors and Actuators B 129 (2008) 364-371.

[38] W.F. Fanga, J.T. Yangb, "A novel microreactor with 3D rotating flow to boost fluid reaction and mixing of viscous fluids", Sensors and Actuators B 140 (2009) 629-642.
[39] Z. Chen, M.R. Bown, B. O. Sullivan, J. M. MacInnes, R.W. K. Allen, M. Mulder, M. Blom, R. van’t Oever, "Performance analysis of a folding flow micromixer", Microfluid Nanofluid (2009) 6:763-774.

[40] P. Garstecki, M J. Fuerstman, M.A. Fischbach, S.K. Sia, G.M. Whitesides, "Mixing with bubbles: a practical technology for use with portable microfluidic devices", Lab on a Chip 6 (2006) 207-212, doi :10.1039/b510843h.

[41] N.T. Nguyen, "Micromixers: Fundamentals, Design and Fabrication", William Andrew, Norwich, NY, USA (2008). 